\title{
Bound states for Laplacian perturbed by varying potential supportedby line in $\mathbb{R}^{3}$
}

\author{
A. S. Bagmutov \\ ITMO University, Kronverkskiy, 49, Saint Petersburg, 197101, Russia \\ bagmutov94@mail.ru
}

DOI 10.17586/2220-8054-2021-12-5-549-552

\begin{abstract}
We investigate a system with attracting $\delta$-potential located along a straight line in 3D. It has constant intensity, except for a local region. We prove the existence of discrete spectrum and construct an upper bound on the number of bound states, using Birman-Schwinger method.
\end{abstract}

Keywords: operator extension theory, singular potential, spectrum.

Received: 17 July 2021

Revised: 10 October 2021

\section{Introduction}

The system under investigation is an exemple of an extensively studied class of models. It can be described as Schroedinger operator in 3D, with singular $\delta$-interactions, supported on a set of zero measure, with codimension two, and often referred to as leaky quantum wires. These models, composed of $\delta$-interaction, concentrated on onedimensional manifolds, are meant to approximate the behaviour of such physical systems, as quantum wires. The operator can be formally written as $-\Delta-\alpha \delta(x-\gamma)$, where the $\gamma$ represents the curve of the wire. A prominent feature of the model is the potential for tunnelling between different parts of the wire, which reflects the behaviour of modelled systems. A region outside of the curve is classically-forbidden, therefore, for bound states, the probability of finding a particle far from the wire declines exponentially and all energies of bound states are negative. An overview of different graph model types, their comparison and some results can be found in [16].

One of the important characteristics of leaky wire systems is the existence and number of bound states, which correspond to localized states of particles. For the investigation of operator spectrum, we use a well-established method, which includes the description of the operator resolvent through a Krein-like formula, which represents the effect of a wire, as a perturbation. This allows the usage of the relevant perturbation theorems. The first appearance of the method, applied to leaky wires model, was in the works $[17,18]$, where the straight line in 3D was considered, and Krein type resolvent formula was used. For a general description of the method dealing with singular interactions, see [2], or for curves in $\mathbb{R}^{3}$ - more recent work [6]. The Krein-like formula was fully described in [3]. There are a number of works, which use this procedure for different types of curves [8,10-12,19,20]. Existing results also include works considering the case of $\mathbb{R}^{2}$ and codimension one $[1,4,5]$ on bent curves or loops [7,9], as well as finite curves and different types of interaction along the curve [13-15]. A model of line-like window in the domain boundary was suggested in [22-25]. It can be considered as a generalization of potential supported by line. For investigation of our system, we will mostly follow the progression of the paper [10].

The system studied in the present paper consists of a straight line in $\mathbb{R}^{3}$, with an attractive $\delta$ potential, generally of constant intensity, except for a finite region, where it is varied.

The paper organized as follows: in section 2 we describe an operator and write a resolvent formula. In section 3 we prove the existence of at least one bound state. In section 4 we construct a Birman-Schwinger operator, and show a method for calculating an upper bound on the number of discrete spectrum points.

\section{Hamiltonian construction}

Let us describe a system. An attractive delta potential is located along a straight line in $\mathbb{R}^{3}$. The intensity is equal to $-(\alpha+\beta(s))$, where $\alpha>0$ is a constant, and $0 \leq \beta(s) \in C(a, b) \backslash\{0\}-$ is a localized function of the distance $s$ along the line, which equals to zero outside of arbitrary finite region $[a, b]$. We choose the 0 point on the line, so that $\beta(0) \neq 0$. Note, that the space for $\beta$ potentially could be expanded.

First, following [10], we describe an operator $-\Delta_{\alpha, \beta}$, which is a self-adjoint extension of the symmetric operator $-\Delta: C_{0}^{\infty}\left(\mathbb{R}^{3} \backslash \Gamma\right) \rightarrow L^{2}\left(\mathbb{R}^{3}\right)$, and then we construct its resolvent. Consider a straight line $\Gamma$, defined by a function $\gamma(s)=(0,0, s): \mathbb{R} \rightarrow \mathbb{R}^{3}$. Let's introduce the shifted curve $\Gamma_{r}$, which corresponds to $\gamma_{r}(s)=(\xi, \eta, s) ;\left(\xi^{2}+\right.$ $\left.\eta^{2}\right)^{1 / 2}=r$. Let $f \in H_{\mathrm{loc}}^{2}\left(\mathbb{R}^{3} \backslash \Gamma\right)$, and $f_{\Gamma_{r}}(s)$, its embedding in $\Gamma_{r} ; r>0$. 
Definition 2.1. A function $f \in H_{\mathrm{loc}}^{2}\left(\mathbb{R}^{3} \backslash \Gamma\right) \cap L^{2}\left(\mathbb{R}^{3}\right)$ belongs to $D\left(-\Delta_{\alpha, \beta}\right)$ if the following conditions are satisfied: 1) The limits

$$
\begin{gathered}
\Xi(f)(s)=-\lim _{r \rightarrow 0} \frac{1}{\ln r} f_{\Gamma_{r}}(s), \\
\Upsilon(f)(s)=-\lim _{r \rightarrow 0}\left(f_{\Gamma_{r}}(s)+\Xi(f)(s) \ln r\right),
\end{gathered}
$$

exist a.e. in $\mathbb{R}$, are independent of the direction $\frac{1}{r}(\xi, \eta)$, and define functions from $L^{2}(\mathbb{R})$,

2) The condition takes place:

$$
2 \pi(\alpha+\beta(s)) \Xi(f)(s)=\Upsilon(f)(s)
$$

Finally we define operator $-\Delta_{\alpha, \beta}: D\left(-\Delta_{\alpha, \beta}\right) \rightarrow L^{2}\left(\mathbb{R}^{3}\right)$ which acts as

$$
-\Delta_{\alpha, \beta} f(x)=-\Delta f(x), x \in \mathbb{R}^{3} \backslash \Gamma .
$$

For description of the $\sigma\left(-\Delta_{\alpha, \beta}\right)$ will be useful the following statement from [1]:

Remark 2.2. A system with one point interaction in $\mathbb{R}^{2}$, has the following spectrum with a single discrete point:

$$
\begin{gathered}
\sigma_{\text {ess }}=[0, \infty), \\
\sigma_{\text {disc }}=\left\{\xi_{1, \alpha}\right\}, \\
\xi_{1, \alpha}=-4 \mathrm{e}^{2(-2 \pi \alpha+\psi(1))},
\end{gathered}
$$

where $\psi(x)=\Gamma^{\prime}(x) / \Gamma(x), \Gamma(x)$ is the Euler Gamma-function, $-\psi(1) \approx 0.5772$ is Euler's number.

Now we will construct a resolvent of $-\Delta_{\alpha, \beta}$. The free resolvent is $R_{z}=(-\Delta-z)^{-1}: L^{2}\left(\mathbb{R}^{3}\right) \rightarrow H^{2}\left(\mathbb{R}^{3}\right), z$ is in resolvent set $z \in \rho(-\Delta)$.

We define the bounded trace map operator of the line:

$$
(\tau \phi)(s)=\phi(s, 0,0): H^{2}\left(\mathbb{R}^{3}\right) \rightarrow L^{2}(\mathbb{R}) .
$$

Its adjoint $\tau^{*}: L^{2}(\mathbb{R}) \rightarrow H^{-2}\left(\mathbb{R}^{3}\right)$ is determined by

$$
\left\langle\tau^{*} h, \omega\right\rangle=(h, \tau \omega), h \in L^{2}(\mathbb{R}), \omega \in H^{-2}\left(\mathbb{R}^{3}\right),
$$

where $\langle\cdot, \cdot\rangle$ stands for duality between $H^{-2}\left(\mathbb{R}^{3}\right)$ and $H^{2}\left(\mathbb{R}^{3}\right)$.

Lets introduce a self-adjoint operator

$$
\begin{gathered}
T_{\kappa} f(s)=\int_{\mathbb{R}}\left(\check{T}_{\kappa}\left(s-s^{\prime}\right)+\frac{1}{2 \pi}(\ln 2+\psi)\right) f\left(s^{\prime}\right) d s^{\prime}=F^{-1}\left[\frac{1}{2 \pi}\left(-\ln \left[\left(p^{2}+\kappa^{2}\right)^{0.5}\right]+(\ln 2+\psi) \delta(p)\right) \hat{f}(p)\right], \\
\check{T}_{\kappa}\left(s-s^{\prime}\right)=-\frac{1}{(2 \pi)^{2}} \int_{\mathbb{R}} \ln \left[\left(p^{2}+\kappa^{2}\right)^{0.5}\right] e^{i p\left(s-s^{\prime}\right)} d p,
\end{gathered}
$$

with the domain $D\left(T_{\kappa}\right)=f \in L^{2}(\mathbb{R}): \int_{\mathbb{R}} \check{T}_{\kappa}\left(s-s^{\prime}\right) f\left(s^{\prime}\right) d s^{\prime} \in L^{2}(\mathbb{R})$, where $-\psi \approx 0.577$ is Euler's number and $F f=\hat{f}$ is a Fourier transform.

Finally, we define self-adjoint operator

$$
Q^{\kappa} f(s)=\left(T_{\kappa}-\beta(s)\right) f(s): D\left(T_{\kappa}\right) \rightarrow L^{2}(\mathbb{R}) .
$$

Now we can construct a resolvent of the main operator, following theorems from [10]:

$$
R_{\beta, \alpha}^{\kappa}=R^{\kappa}-R^{\kappa *} \tau^{*}\left(Q^{\kappa}-\alpha\right) \tau R^{\kappa} .
$$

\section{Existence of bound states}

Let us denote as $\beta_{0}>0$ and $\beta_{w}>0$, any two small enough numbers, so that $\beta(x)>\beta_{0} ; \forall|x|<\beta_{w} / 2$, and $\sup \beta(s)=\beta_{s}$.

First, lets notice that $\beta(s)$, being a local perturbation of a potential, does not change essential spectrum of the main operator:

$$
\sigma_{\text {ess }}\left(-\Delta_{\alpha, \beta}\right)=\sigma_{\text {ess }}\left(-\Delta_{\alpha}\right)=\left[\xi_{1, \alpha}, \infty\right)=\left[-4 e^{2(-2 \pi \alpha+\psi)}, \infty\right) .
$$

Using the momentum representation of $T_{\kappa}$ and locality of $\beta(s)$, we can get

$$
\begin{gathered}
\sigma_{e s s}\left(T_{\kappa}\right)=\sigma_{e s s}\left(Q^{\kappa}\right)=\left(-\infty, s_{\kappa}\right], \\
s_{\kappa}=\frac{1}{2 \pi}\left(\psi(1)-\ln \frac{\kappa}{2}\right) .
\end{gathered}
$$

Note that $s_{\kappa}=\alpha$ corresponds to $-\kappa^{2}=\xi_{1, \alpha}$ of the main operator. 
Lemma 3.1. $\sup \sigma\left(Q^{\kappa}\right)=\sup \sigma\left(T_{\kappa}-\beta(s)\right)>s_{\kappa}$.

Proof. The lemma is equivalent to

$$
\left(Q^{\kappa} \phi, \phi\right)-s_{\kappa}(\phi, \phi)>0
$$

for any $\phi \in D\left(Q^{\kappa}\right)$. Let $\phi \in C_{0}^{\infty}(\mathbb{R})$, such that $\exists C>0, \delta>0: \phi(s)>C,|s|<\delta$ and we will use $\phi_{\lambda}(s)=$ $\lambda^{0.5} \phi(\lambda s) ; \lambda>0$, notice, still $\phi(\lambda s)>C,|s|<\delta$, and $\left\|\phi_{\lambda}\right\|=\|\phi\|$. We get

$$
\frac{1}{2 \pi} \int_{\mathbb{R}} \ln \left[\left(1+\frac{\lambda^{2} u^{2}}{\kappa}\right)^{0.5}\right]|F \phi(u)|^{2} d u+\lambda \int_{\mathbb{R}} \beta(s)|\phi(\lambda s)|^{2} d s>0,
$$

where the first term can be expanded as $-\frac{1}{4 \pi}\left(\frac{\lambda}{\kappa}\right)^{2} \int_{\mathbb{R}} u^{2}|F \phi(u)|^{2} d u+\mathcal{O}\left(\lambda^{4}\right)$, and the second one:

$\lambda \int_{\mathbb{R}} \beta(s)|\phi(\lambda s)|^{2} d s>\lambda \int_{-\delta}^{\delta} \beta(s) C^{2} d s>2 \delta C \beta_{0} \beta_{w}$. So for the $\lambda$ small enough, the second term sways the sum in the positive direction.

Lemma 3.2. The function $\kappa \rightarrow Q^{\kappa}$ is continuous in the norm operator sense on $\left(\kappa_{0}, \infty\right)$, and

$$
\lim _{\kappa \rightarrow \infty} \sup \sigma\left(Q^{\kappa}\right)=-\infty \text {. }
$$

Proof. Function, $\kappa \rightarrow T_{\kappa}$ is continuous in the norm-operator sense:

$$
\left\|\left(T_{\kappa}-T_{\kappa}^{\prime}\right) f\right\|=\frac{1}{4(2 \pi)^{3}} \int_{\mathbb{R}}\left(\ln \frac{p^{2}+\kappa^{2}}{p^{2}+\kappa^{\prime 2}}\right)^{2}|F f(p)|^{2} d p \leq \frac{1}{4(2 \pi)^{3}}\left(\ln \frac{\kappa}{\kappa^{\prime}}\right)^{2}\|f\|^{2} \underset{\kappa^{\prime} \rightarrow \kappa}{\longrightarrow} 0
$$

and $\beta(s)$ is independent of $\kappa$, so $Q^{\kappa}=T_{\kappa}-\beta(s)$ is continuous. Limit (2) follows from:

$$
\begin{gathered}
\left(Q^{\kappa} f, f\right)=\frac{1}{(2 \pi)^{3 / 2}} \int_{\mathbb{R}}\left(-\ln \sqrt{p^{2}+\kappa^{2}}+\ln 2+\psi(1)\right)|\hat{f}(p)|^{2} d p+(\beta(s) f, f) \leq \\
\leq \frac{1}{(2 \pi)^{3 / 2}}\left(-\ln \frac{\kappa}{2}+\psi(1)\right)\|f\|^{2}+\beta_{s} *\|f\|^{2} .
\end{gathered}
$$

Now, analogous to theorem 5.6 of [10], we are ready to prove the existence of at least one bound state.

Theorem 3.1. The operator $-\Delta_{\alpha, \beta}$ has at least one isolated eigenvalue in $\left(-\infty, \xi_{1, \alpha}\right)$.

Proof. The addition of localized potential $\beta(s)$ can alter only discrete part of a spectrum, i. e. for the main operator - the part that lies in $\left(-\infty, \xi_{1, \alpha}\right)$ and for $Q^{\kappa}$, it lies in $\left(s_{\kappa}, \infty\right)$. Then by Lemma 3.1, there exists at least one discrete point of spectrum for $Q^{\kappa}, \lambda(\kappa)$. By Lemma 3.2, $\lambda(\kappa)$ is continuous and $\lambda \rightarrow-\infty$ as $\kappa \rightarrow \infty$. Therefore $\exists \kappa^{\prime}>$ $\left|\xi_{1, \alpha}\right|^{0.5}: \lambda\left(\kappa^{\prime}\right)=\alpha$. The point $-\kappa^{\prime 2}$ is a pole of resolvent (1), and therefore is an eigenvalue of the main operator.

\section{Upper bound on number of bound states}

Now we can utilize the Birman-Schwinger method (see [21]) to get an upper bound on the number of bound states for the main operator.

Let's construct a Birman-Schwinger operator, which we will use to count eigenvalues.

$$
\begin{aligned}
Q^{\kappa} f-\alpha f & =0, \\
T_{\kappa} f-\alpha f-\beta(s) f & =0, \\
K_{\kappa} f \equiv\left(T_{\kappa}-\alpha\right)^{-1}(\beta(s) f) & =f .
\end{aligned}
$$

Here, if $f$ is an eigenvector of $Q^{\kappa}$, corresponding to the eigenvalue $\lambda_{Q}=\alpha$, then it is an eigenvector of $K_{\kappa}$, corresponding to the eigenvalue $\lambda_{K}=1$. Also, note that eigenvalues of $Q^{\kappa}$ are monotonically decreasing as functions of $\kappa$, and so are eigenvalues of $K_{\kappa}$, therefore, if we fix $\kappa$, for each discrete point of spectrum of the main operator $-\Delta_{\alpha, \beta}$, there is a corresponding eigenvalue of $Q^{\kappa}$, bigger then $\alpha$, and corresponding eigenvalue of $K_{\kappa}$, bigger then 1. Summing up all these eigenvalues of $K_{\kappa}$, we get an upper bound on the number of eigenvalues $\lambda\left(Q^{\kappa}\right)>\alpha$ and number of discrete points of $-\Delta_{\alpha, \beta}$, smaller then $-\kappa^{2}$. To cover all $\sigma_{\text {disc }}\left(-\Delta_{\alpha, \beta}\right)$, we approach the $\sigma_{e s s}\left(-\Delta_{\alpha, \beta}\right)$ threshold and get the final bound:

Proposition 4.1. The number of points in $\sigma_{d i s c}\left(-\Delta_{\alpha, \beta}\right)$, has the following upper bound

$$
\# \sigma_{d i s c}\left(-\Delta_{\alpha, \beta}\right) \leq \lim _{-\kappa^{2} \uparrow \xi_{1, \alpha}} \int_{\mathbb{R}^{2}}\left(\int \frac{e^{2 \pi i(s-p) w}}{-\ln \sqrt{w^{2}+\kappa^{2}}+\left(-\ln \frac{\kappa}{2}+\psi(1)-\alpha\right) \delta(w)} d w \beta(p)\right)^{2} d s d s^{\prime} .
$$




\section{Acknowledgements}

The reported study was funded by RFBR, project number 20-31-90050.

\section{References}

[1] Albeverio S., Gesztesy F., Høegh-Krohn R., Holden H. Solvable Models in Quantum Mechanics, Springer, Heidelberg, 1988.

[2] Brasche J., Exner P., Kuperin Yu.A., Seba P. Schrödinger operator with singular interactions. J. Math. Anal. Appl., 1994, 184, P. 112-139.

[3] Posilicano A. A Krein-like formula for singular perturbations of self-adjoint operators and applications. J. Funct. Anal., 2001,183 , P. 109-147.

[4] Shondin Yu. On the semiboundedness of delta-perturbations of the Laplacian on curves with angular points. Theor. Math. Phys., 1995, 105, P. 1189-1200.

[5] Behrndt J., Langer M. Boundary value problems for elliptic partial differentialoperators on bounded domains. J. Funct. Anal., 2007, 243, P. 536-565.

[6] Behrndt J., Frank R.L., Kuhn Ch., Lotoreichik V., Rohleder J. Spectral theory for Schroedinger operators with $\delta$-interactions supported on curves in $\mathbb{R}^{3}$. Ann. H. Poincar'e, 2017, 18, P. 1305-1347.

[7] Exner P., Ichinose T. Geometrically induced spectrum in curved leaky wires. J. Phys., 2001, A34, P. 1439-1450.

[8] Exner P., Kondej S. Gap asymptotics in a weakly bent leaky quantum wire. J. Phys., 2015, A48, P. 495301.

[9] Exner P., Yoshitomi K. Asymptotics of eigenvalues of the Schroedinger operator with a strong delta-interaction on a loop. J. Geom. Phys., 2002, 41, P. 344-358.

[10] Exner P., Kondej S. Curvature-induced bound states for a delta interaction supported by a curve in $R^{3}$. Ann. H. Poincare, 2002, 3, P. 967-981.

[11] Exner P., Kondej S. Hiatus perturbation for a singular Schrödinger operator with an interaction supported by a curve in $\mathbb{R}^{3}$. J. Math. Phys., 2008, 49, P. 032111.

[12] Exner P., Kondej S. Scattering by local deformations of a straight leaky wire. J. Phys., 2005, A38, P. $4865-4874$.

[13] Exner P., Jex M. Spectral asymptotics of a strong $\delta^{\prime}$ interaction on a planar loop. J. Phys., 2013, A46, P. 345201.

[14] Exner P., Tater M. Spectra of soft ring graphs. Waves Random Media, 2003, 14, P. S47-S60.

[15] Behrndt J., Langer M., Lotoreichik V. Schroedinger operators with $\delta$ and $\delta^{\prime}$-potentials supported on hypersurfaces. Ann. Henri Poincar'e, 2013, 14, P. 385-423.

[16] Exner P. Leaky quantum graphs: a review, Analysis on Graphs and its Applications. Selected papers based on the Isaac Newton Institute for Mathematical Sciences programme, Cambridge, UK, 2007, Proc. Symp. Pure Math., 2008, 77, P. $523-564$.

[17] Kurylev Ya. Boundary conditions on curves for the three-dimensional Laplace operator. Journal of Soviet Mathematics, 1983, 22(1), P. 10721082.

[18] Blagovescenskii A.S.,Lavrent'ev K.K. A three-dimensional Laplace operator with a boundary condition on the real line (in Russian). Vestn.Leningr.Univ., Math. Mekh. Astron., 1977, 1, P. 9-15.

[19] Exner P., Kondej S. Strong coupling asymptotics for Schrodinger operators with an interaction supported by an open arc in three dimensions. Rep. Math. Phys., 2016, 77, P. 1-17.

[20] Eremin D.A., Ivanov D.A., Popov I.Yu. Regular Potential Approximation for delta-Perturbation Supported by Curve of the Laplace-Beltrami Operator on the Sphere. Zeitschrift fur Analysis und Ihre Anwendungen, 2012, 31(2), P. 125-137.

[21] Reed M., Simon B. Methods of Modern Mathematical Physics. IV. Analysis of Operators. Academic Press, New York, 1978.

[22] Pavlov B.S., Popov I.Y. Model of diffraction on an infinitely-narrow slit and the theory of extensions. Vestnik Leningrad. Univ. Ser. Mat., Mekh., Astr., 1983, 4, P. 36-44

[23] Popov I.Yu. The extension theory and localization of resonances for the domain of trap type. Matematicheskii sbornik, 1990, 181(10), P. 13661390. English: Mathematics of the USSR-Sbornik, 1992, 71(1), P. 209-234.

[24] Popov I.Y. The resonator with narrow slit and the model based on the operator extensions theory. J. Math. Phys., 1992, 33(11), P. 3794-3801.

[25] Popov I.Yu. Helmholtz resonator and the operator extension theory in a space with an indefinite metrics. Matematicheskii sbornik, 1992, 183(3), P. 2-38. English translation in Russian Acad. Sci. Sb. Math., 1993, 75(2), P. 285-315. 